\title{
THE HUGHES PROBLEM AND OTHERS
}

\author{
I. D. MACDONALD ${ }^{1}$
}

(Received 28 March 1969)

\section{To Bernhard Hermann Neumann on his 60th birthday}

Communicated by G. E. Wall

1.

Let $X$ be a finite $p$-group with the anti-Hughes property

$$
\left|X: H_{p}(X)\right|=p^{2}, \quad H_{p}(X)>1, \quad d(X)=2 .
$$

(Notation is set forth in Section 2.) Any element of a finite $p$-group $G$ not in $H_{p}(G)$ has order $p$, hence either $G / H_{p}(G)$ is non-abelian or $H_{p}(G) \geqq \Phi(G)$. Therefore $X$ has the property

$$
H_{p}(X)=\Phi(X)
$$

all its elements of maximal order lie in $\Phi(X)$.

Now let $G$ be a finite $p$-group such that $G>H_{p}(G)$; then $G$ is generated by a set of elements of order $p$, viz. the complement of $H_{p}(G)$, so that $\Phi(G)=\delta(G)$. Our assumption about $X$ leads to

$$
H_{p}(X)=\delta(X) \text {; }
$$

all its elements of maximal order lie in $\delta(X)$.

Suppose ${ }^{2}$ in addition that $H_{p}(G) \leqq \delta(G)$. Choose a normal subgroup $N$ of $G$ which has index $p$ in $V_{p}(G)$, so that

$$
1 \leqq N<V_{p}(G) \leqq H_{p}(G) \leqq \delta(G)<G .
$$

Then $\delta(G / N)$ has exponent $p^{2}$ while $(G / N) / \zeta(G / N)$ has exponent $p$ (being isomorphic to a factor group of $\left.G / V_{p}(G)\right)$. Our assumption about $X$ now gives:

(P4) There is a finite p-group $Y$ for which $\exp (\delta(Y))$ does not divide $\exp (Y / \zeta(Y))$.

1 I thank the University of Dundee for its generosity in respect of finance and hospitality.

2 The substance of this paragraph was conveyed to me by G. E. Wall, who attributed it to J. L. Alperin. Note that the symbol $\delta$ occurs in the argument in a formal manner only, so that $\delta(G)$ could be interpreted as (for instance) $\Phi(G)$. 
In fact this group $Y$ has a further property that is worth mentioning. Because $H_{p}(G / N) \leqq H_{p}(G) / N$ we have $\left|Y: H_{p}(Y)\right| \geqq p^{2}$ while of course $1<H_{p}(Y) \leqq \delta(Y)$. Let $y_{0}$ be an element of order $p^{2}$ in $\delta(Y)$ and let $x_{0}$ be an element outside $H_{p}(Y)$. Since both $x_{0}$ and $x_{0} y_{0}$ have order $p$ it follows that $g p\left\{x_{0}, y_{0}\right\}$ is not regular. Hence

(P5) There is a finite p-group $Y$ which satisfies

(a) $Y$ has a central subgroup $Z$ of order $p$ such that $Y \mid Z$ is regular; and

(b) there are elements $x_{0}$ in $Y$ and $y_{0}$ in $\delta(Y)$ for which $g p\left\{x_{0}, y_{0}\right\}$ is not regular.

Whether a group $X$ with (P1) exists or not appears to be an open question. This is unfortunate because (P2), (P3), (P4) and (P5) have all attracted attention in recent years. Thus (P3) is discussed by Kappe in [4], pp. $362-363$. Hobby's nearly regular $p$-groups in $[3]$ are defined by (a) and the negation of (b) in (P5), and the existence of $Y$ (and therefore of $X$ ) would solve negatively a problem of his. Finally (P2) and (P4) seem to have been subjects of unrecorded speculation and conjecture.

Nevertheless some progress has been made along similar lines, mainly by the construction of groups $W$ and $M$. The former, presented by Wall in [7], is a finite 5-group of exponent 25 with $\left|W: H_{5}(W)\right|=25$ and $d(W)>2$; both $H_{5}(W) \leqq \delta(W)$ and the analogues of (P4) and (P5) appear to fail. The finite 2-group $M$ in [5] has $d(M)=2$ and $\delta(M)<H(M)<\Phi(M)$ where $H(M)$ is the subgroup generated by the elements of order 8 , so that there is a property like (P2), but nothing like (P3), (P4), and (P5). We note that the construction of a finite $p$-group $G$ with $d(G)>2$ and having inside $\Phi(G)$ all its elements of maximal order seems to be a difficult matter.

We have now said enough to generate some interest in the existence of $X$ with (P1) and to suggest that this is a problem of considerable significance in the theory of finite $p$-groups. In the present note a small contribution is made towards the solution; it will be shown that such a group as $X$ must be nilpotent of class at least $2 p$. The original aim was to construct and investigate an example of $X$, but our result shows that it is hardly feasible to do this by means of a free presentation. Since it is well known that there is no $X$ with $p<5$ (and indeed Hobby proves that there is no $Y$ with $p<5$ ), the most favourable remaining possibility is $p=5$ and class 10 , too complicated a situation for elementary methods.

\section{2.}

Commutator notation will be standard left-normed. Thus $\left(x_{1}, x_{2}\right)$ means $x_{1}^{-1} x_{2}^{-1} x_{1} x_{2}$, and $x_{1}^{x_{2}}$ means $x_{2}^{-1} x_{1} x_{2}$. Let $m_{1}, \cdots, m_{n}$ with $n \geqq 2$ be positive integers. Then the left-normed commutator $\left(x_{1}, m_{2} x_{2}, \cdots, m_{n} x_{n}\right)$ is defined 
inductively as $\left(\left(x_{1}, m_{2} x_{2}, \cdots,\left(m_{n}-1\right) x_{n}\right), x_{n}\right)$ when $m_{n}>1$ and as $\left(\left(x_{1}, m_{2} x_{2}, \cdots, m_{n-1} x_{n-1}\right), x_{n}\right)$ when $m_{n}=1$ and $n>2$, with $\left(x_{1}, 1 x_{2}\right)=$ $\left(x_{1}, x_{2}\right)$. The subgroup generated by the subset $S$ of the group $G$ will be written as $g p\{S\}$. When $S=\left\{\left(x_{1}, \cdots, x_{n}\right): x_{1} \in G, \cdots, x_{n} \in G\right\}$ then $g p\{S\}$ is $\gamma_{n}(G)$, with $\gamma_{1}(G)=G$. We define $\delta(G)$ to be $\gamma_{2}(G)$. The group $G$ is said to be nilpotent of class $c$ if $\gamma_{\mathrm{c}+1}(G)=1$ for some $c \geqq 0$.

For any group $G$ and any positive integer $n$ we define $V_{n}(G)$ or $G^{n}$ to be $g p\left\{x^{n}: x \in G\right\}$, and if $G$ is finite then we define its exponent $\exp (G)$ to be the least positive $n$ such that $V_{n}(G)=1$. The Frattini subgroup $\Phi(G)$ of the finite group $G$ is the intersection of its maximal subgroups, which in the case of a $p$-group is just $g p\left\{V_{p}(G), \delta(G)\right\}$. Also for a finite $p$-group $G$ the minimal number of generators $d(G)$ is given by $|G: \Phi(G)|=p^{d(G)}$; we use the symbol $|G: H|$ to denote the index of the subgroup $H$ in the group $G$.

We write $\zeta(G)$ for the centre of $G$. The Hughes subgroup $H_{p}(G)$ of the group $G$ for the prime $p$ is defined as the subgroup generated by all the elements of $G$ that do not have order $p$.

We note the identity

$$
(u v, x y)=(u, y)^{v}(v, y)(u, x)^{v y}(v, x)^{y} .
$$

We shall use some elementary results about commutator collection, details of which may be found in [2].

3.

Lемма. If $G$ is a finite p-group such that $V_{p}(G) \leqq \zeta(G)$ then $\gamma_{3}(G)^{p} \leqq \gamma_{2 p+1}(G)$.

Proof. Put $H=g p\left\{y, y^{x}\right\}=g p\{y,(y, x)\}$ where $x, y$ are for the moment arbitrary elements of $G$. Since $y^{p}=\left(y^{x}\right)^{p}$ commutator collection gives

$$
(y, x)^{p}=\left(y^{-1} y^{x}\right)^{p} \in \gamma_{2}(H)^{p} \gamma_{p}(H) .
$$

Now suppose that $y \in \gamma_{k}(G)$ for some $k \geqq 1$. It follows that

$$
(y, x)^{p} \in \gamma_{2 k+1}(G)^{p} \gamma_{p k+1}(G),
$$

and as a result of this we have

Hence

$$
\gamma_{k+1}(G)^{p} \leqq \gamma_{2 k+1}(G)^{p} \gamma_{p k+1}(G)
$$

$$
\gamma_{k+1}(G)^{p} \leqq \gamma_{p k+1}(G)
$$

and the case $k=2$ gives the lemma.

Theorem. If the finite p-group $G$ has $\left|G: H_{p}(G)\right| \geqq p^{2}, V_{p}(G) \leqq \zeta(G)$ and $\gamma_{2 p}(G)=1$, then $\left(H_{p}(G) \cap \delta(G)\right)^{p}=1$. 
Proof. We suppose that the finite $p$-group $G$ satisfies the hypothesis of the theorem. Let $y$ be an element in $H_{p}(G) \cap \delta(G)$, and $x$ be an element not in $H_{p}(G)$; and consider $y^{-p} x^{-p}(x y)^{p}$. Since $x^{p}=(x y)^{p}=1$ this element is just $y^{-p}$; on the other hand commutator collection shows that it lies in $\gamma_{3}(G)^{p} \gamma_{p+1}(G)$, which is $\gamma_{p+1}(G)$ in view of the lemma. More precisely, $y^{-p}$ is a power product of commutators each having at least $p$ entries from $\{y, x\}$. Note that $\gamma_{p}(G)$ is abelian since $\gamma_{2 p}(G)=1$.

Because $\left|G: H_{p}(G)\right| \geqq p^{2}$ we can find elements $x_{1}, x_{2}$ in $G$ such that if $x_{1}^{\alpha} x_{2}^{\beta} \in H_{p}(G)$ with $0 \leqq \alpha, \beta<p$ then $\alpha=\beta=0$. Substituting $x_{1}^{\alpha} x_{2}^{\beta}$ for $x$ in the expression for $y^{-p}$ enables us to arrive at another expression for $y^{-p}$, this time a power product of commutators having at least $p$ entries from $\left\{y, x_{1}^{\alpha}, x_{2}^{\beta}\right\}$, and at least one of these entries is $y$.

Now lemma $\mathrm{H} 2$ of [6] and the remarks following that lemma give some information about the result of expanding a commutator whose $r$ entries are just $\left\{b_{1}, \cdots, b_{i-1}, b_{i}^{\alpha}, b_{i+1}, \cdots, b_{r}\right\}$. It becomes $v_{0}^{f_{0}(\alpha)} v_{\mathbf{1}}^{f_{1}(\alpha)} \cdots$, where each $v_{j}$ is a commutator with $r_{j}$ entries from $\left\{b_{1}, \cdots, b_{i}, \cdots, b_{r}\right\}, r_{j} \geqq r$, and $f_{j}(\alpha)$ is a polynomial with coefficients integers modulo $p$ and of degree at most $r_{j}-r+1$ in $\alpha$.

We carry out this transformation repeatedly on all our commutators with entries from $\left\{y, x_{1}^{\alpha}, x_{2}^{\beta}\right\}$. After a sufficient number of operations we obtain commutators with at least $p$ entries from $\left\{y, x_{1}, x_{2}\right\}$, at least one entry being $y$. Since $\gamma_{2 p}(G)=1$, and $y \in \gamma_{2}(G)$, there will be at most $2 p-3$ entries from $\left\{x_{1}, x_{2}\right\}$ in each commutator. The polynomials occurring as exponents of the commutators will therefore have total degree not exceeding $2 p-3$ in $\{\alpha, \beta\}$.

There results an expression

$$
y^{-p}=\prod c_{i j}^{\alpha^{i} \beta^{j}}
$$

the $c_{i j}$ are power products of commutators with at least $p$ entries from $\left\{y, x_{1}, x_{2}\right\}$, and the product is taken over non-negative $\mathrm{i}, j$ with

$$
1 \leqq i+j \leqq 2 p-3 \text {. }
$$

We now use the fact that $\alpha$ and $\beta$ can take all values from 0 to $p-1$, except $\alpha=\beta=0$, to "factorise" the above expression; this process is explained in detail and quite generally in $[\mathbf{1}]$. We obtain

$$
y^{-p}=c_{p-1,0}^{\alpha,-1} c_{0, p-1}^{\beta^{p-1}} .
$$

On putting $\alpha=1, \beta=0 ; \alpha=0, \beta=1 ; \alpha=1, \beta=1$ in succession we find that $y^{p}=1$. Therefore $\left(H_{p}(G) \cap \delta(G)\right)^{p}=1$.

Corollary 1. If the finite p-group $G$ has $H_{p}(G) \leqq \delta(G)$ and $\gamma_{2 p}(G)=$ ] then $H_{p}(G)=1$. 
Proof. We may suppose that $V_{p}(G) \leqq \zeta(G)$, for otherwise we can replace $G$ by a factor group of itself having all the desired properties; the details of this process are suppressed, being very similar to the transition from $X$ to $Y$ in Section 1 . The corollary then follows easily from the theorem.

CoROLlary 2. If the finite $p$-group $G$ has $H_{p}(G) \leqq \Phi(G)$ and $\gamma_{2 p}(G)=1$ then $H_{p}(G)=1$.

Proof. Recall that if $G>H_{p}(G)$ then $\Phi(G)=\delta(G)$.

Corollary 3. If the finite p-group $G$ has $\left|G: H_{p}(G)\right| \geqq p^{2}, d(G)=2$ and $\gamma_{2 p}(G)=1$ then $H_{p}(G)=1$.

Proof. We have $d\left(G / H_{p}(G)\right)=2$ since $d(G)=2$ and $G / H_{p}(G)$ has exponent $p$. Therefore, in $G / H_{p}(G)$, the Frattini subgroup has index $p^{2}$. But the fact that $\Phi(G)=\delta(G)$ then gives $\left|G: \delta(G) H_{p}(G)\right|=p^{2}$, as well as $|G: \delta(G)|=p^{2}$. We conclude that $H_{p}(G) \leqq \delta(G)$, and so the results above yield Corollary 3.

(Added in proof.) The methods of this note serve to prove the following theorem: in a finite $p$-group of class $2 p-2$ the Hughes conjecture is true. Details will appear in the first issue of the Nigerian Journal of Mathematics.

\section{References}

[1] Warren Brisley, 'On varieties of metabelian p-groups, and their laws', J. Austral. Math. Soc. 7 (1967), $64-80$.

[2] Marshall Hall Jr., The Theory of Groups (Macmillan, New York, 1959).

[3] C. R. Hobby, 'Nearly regular p-groups', Canad. J. Math. 19 (1967), 520-522.

[4] Wolfgang P. Kappe, 'Properties of groups related to the second centre', Math. Z. 101 (1967), 356-368.

[5] I. D. Macdonald, 'Some examples in the theory of groups', to appear in the A. J. Macintyre Memorial Volume, University of Cincinnati, 1969.

[6] Ruth Rebecca Struik, 'On nilpotent products of cyclic groups', Canad. J. Math. 12 (1960), $446-461$.

[7] G. E. Wall, 'On Hughes' $H_{p}$ problem', Proc. Internat. Cont. Theory of Groups (Canberra, 1965), pp. 357-362. (Gordon and Breach, New York, 1967).

The University of Dundee

Dundee, Scotland 